\title{
SUSTAINABLE LAND-USE DECISION MAKING FROM THE GEOLOGICAL POINT OF VIEW: AN EXAMPLE FOR THE USE OF GEO-RESOURCES IN A METROPOLITAN AREA
}

\author{
Oswald Marinoni \\ Stefan Lang \\ Christian Lerch \\ Andreas Hoppe \\ Technische Universität Darmstadt \\ Institute for Applied Geosciences \\ Geo-Resources and Geo-Hazards \\ Schnittspahnstraße 9 \\ D-64287 Darmstadt - Germany \\ marinoni@geo.tu-darmstadt.de
}

Keywords: AHP, land-use, conflict map, geo-resources, GIS

Summary: Sand and gravel are important raw materials which are needed for many civil engineering projects. Due to economic reasons, sand and gravel pits are frequently located in the periphery of metropolitan areas which are often subject to competing land-use interests. As a contribution to land-use conflict solving, the Analytic Hierarchy Process (AHP) is applied within a Geo-Information-System (GIS) environment. Two AHP preference matrix scenario constellations are evaluated and their results are used to create a land-use conflict map.

\section{Introduction}

Geo-resources like sand and gravel are important raw materials which are mainly needed for concrete production in civil engineering projects. This makes them valuable especially in metropolitan areas where construction activities are frequent. In contrast to their meaning for construction purposes, their economic value is relatively low so that their transport over greater distances is normally not profitable in most cases. To be run economically, sand and gravel pits are therefore frequently located in or very close to metropolitan areas so that transportation costs can be restricted to a minimum.

From the geological point of view, sand and gravel bodies often represent valuable groundwater bodies which may serve as sources for potable water. To preserve these resources for future generations, they should therefore remain untouched as far as possible. However, due to the current need for sand and gravel and economic reasons (e.g. number of jobs), the exploitation of these resources should be made possible to a minimum amount. Other considerations may concentrate on ecological impacts or recreational values. Therefore raw material exploitation in or close to metropolitan areas implies a high potential for conflicts. If the location for a new exploitation site has to be determined, a variety of criteria have to be considered. In this contribution, the Analytic Hierarchy Process (AHP) is used to create suitability maps for sand and gravel mining in the vicinity of Frankfurt, one of Germany's most important economic centers, where large amounts of sand and gravel were accumulated inside a subsidence river basin during the ice age period. 


\section{Spatial decision support with the AHP}

To effectively handle spatial data, it is state of the art to use GIS technologies. The fact that many GIS are equipped with excellent visualisation interfaces increases the decision-maker's comprehension of the spatial information that is involved in the problem being addressed (Tkach and Simonovic 1997). Besides their great visualisation capabilities, GIS offer comfortable functionalities with respect to spatial querying and spatial data analysis. GIS have therefore been repeatedly combined with various multi-criteria decision support methodologies in the last years (Carver, 1991; Pereira and Duckstein, 1993; Jankowski et al., 1997; Malczewski, 1999; Marinoni, 2005) whereby the AHP was also frequently used (Eastman et al. 1998; Dai et al. 2001; Araújo and Macedo 2002; Marinoni 2004). Due to the possibility to develop criteria weights, the AHP has already been called one of the most promising techniques (Eastman et al. 1998). The combination of GIS and multi-criteria decision support methods is commonly referred to as Spatial Decision Support System (SDSS). The following figure 1 gives the context in which the AHP is used within a land-use decision process.

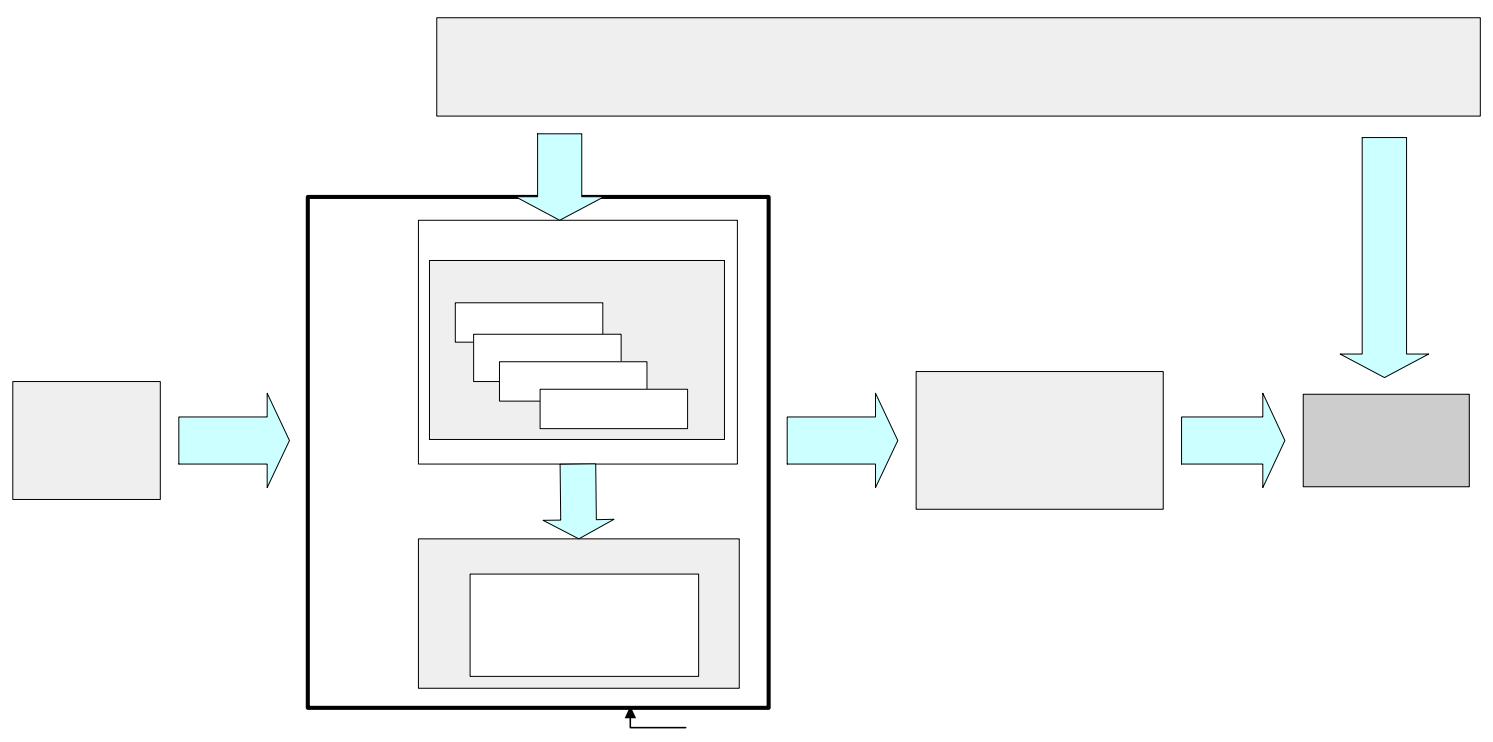

Figure 1: Workflow of a land-use decision process using a SDSS.

\section{Case study - determination of potential sand and gravel pit locations}

\subsection{Description of the project area}

The project area is located in the southeast of Frankfurt (see figure 2). Long-term subsidence has allowed the accumulation of substantial river deposits containing large amounts of gravel and sand during the ice age period. Thus intensive mining activities are presently run in this area. Due to the proximity to the metropolitan area as well as the suitability of the mining areas for several different purposes, there are considerable conflicts of land-use interests.

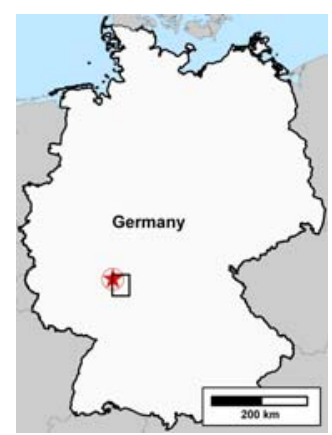

Figure 2: Location of the study area (square) in the southeast of Frankfurt. 


\subsection{Definition of criteria}

Statements about the suitability of an area for a specific land-use depend on the criteria which have to be defined first. The objective here is to determine the suitability of a region for sand and gravel mining. As already stated in section 1 , the use of these geo-resources implies quite a few conflicts which result from the following facts

(i) Since sand and gravel have a good hydraulic conductivity, they frequently form groundwater-bodies which are used as sources for potable water. Sand and gravel are therefore considered to have a high hydro(geo)logical value.

(ii) Though there is a high demand for sand and gravel, their economic value is fairly low. An exploitation is profitable only if the transport costs are low and the exploitable volume is large.

(iii) As sustainability plays a major role, the area used for raw material extraction should be as small as possible. Small areas of raw material exploitation are favored if the thickness of a deposit is high.

(iv) Transport of the raw material coincides with a higher noise level through a higher amount of truck traffic. Moreover, the mining itself can be noisy and dust may pollute the environment next to the pit.

(v) If raw material extraction is allowed, the landscape is changed sustainably.

The mentioned conflicts can be taken into account if the criteria for the suitability study are carefully chosen. For the case study the following criteria were selected:

(i) To take account of the high hydro(geo)logical value, sand and gravel mining should only be allowed in zones with no or at least a low level of groundwater protection. In Germany, these zones are defined by the regional authorities.

(ii) To enable sand and gravel enterprises to work economically, sand and gravel mining should be allowed within a metropolitan area. However, to keep dust pollution and noise levels as low as possible, a minimum distance of 1000 meters to settlements should be kept..

(iii) To support mining profitability, the thickness of the overburden (the material on top of the deposit) should be as small as possible, while the thickness of the exploitable raw material should be as large as possible.

(iv) Soil productivity was also chosen to play a significant role. Areas with soils of high agricultural productivity should preferably be avoided.

The fact that sand and gravel mining does sustainably change a landscape is a very important concern. But this sustainable change of the landscape cannot be avoided. However, it has lately been recognized that areas of former sand and gravel may serve as "second hand biotopes", where the biodiversity may be greater than before the mining period (BfN 2001). Moreover, some former pits are now used as recreational areas which are usually well accepted by the local population.

\subsection{GIS based evaluation}

To take advantage of GIS-technology all criteria were introduced to a GIS as raster datasets. However, many criteria are measured in different dimensions or in different value ranges which makes it necessary to bring them to one common scale. In this case study, all criteria were reclassified which is an easy task with a GIS. The number of classes was derived from the available classes of soil productivity (table 1). There is a total number of 9 classes (from very low to very high) for soil productivity. However, soils with "very low" and "very low to low" productivity were not found in the study area so that the total number of classes was reduced to 7 covering a value range from 0 to 6 respectively. Please note that the soils with the highest productivity obtain the lowest (and therefore worst) class value. This is due to the fact that areas with a high productivity of soils should be avoided according to criterion (iv) (see above). The regionalized map of the assigned class values is shown in figure 3. 
Table1: Classes of soil productivity encountered in the study area.

\begin{tabular}{ccc}
\hline \hline Soil productivity & Value & Class value \\
\hline Very low & 1.00 & not encountered in \\
Very low - low & 1.50 & study area \\
Low & 2.00 & $\mathbf{6}$ \\
Low - medium & 2.50 & $\mathbf{5}$ \\
Medium & 3.00 & $\mathbf{4}$ \\
Medium - high & 3.50 & $\mathbf{3}$ \\
High & 4.00 & $\mathbf{2}$ \\
High - very high & 4.50 & $\mathbf{1}$ \\
Very high & 5.00 & $\mathbf{0}$ \\
\hline \hline
\end{tabular}

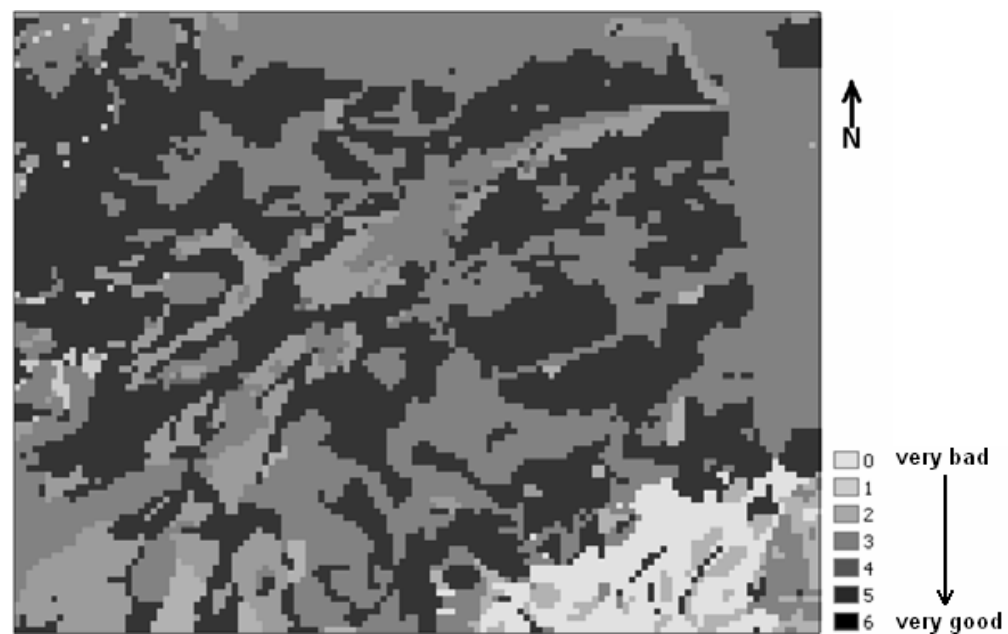

Figure 3: Class values for soil productivity. Low class values are considered bad for the intended land-use, high class values are considered good (for more details on the class values see table 1).

The distance from existing settlements should exceed 1000 meters. To visualize the areas which fulfil this criterion, buffer zones were calculated around the existing settlements. Areas inside this $1000 \mathrm{~m}$ buffer were not considered for further sand and gravel mining suitability considerations. Distances between 1000 meters and 4800 meters were linearly divided into 7 classes (class values ranging from 0 to 6 ). The greater the distance to a settlement, the higher (and better) the assigned class value (figure 4).

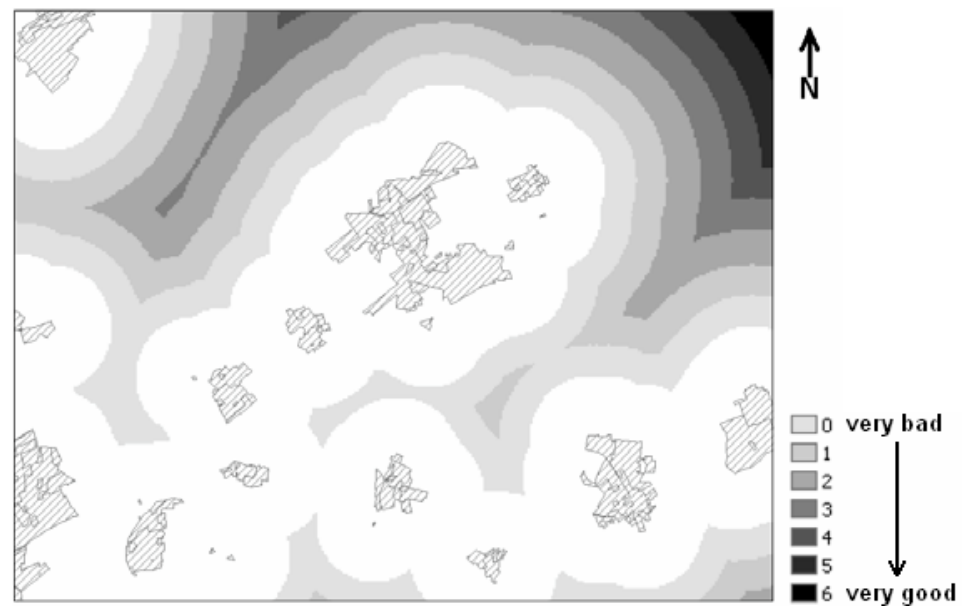

Figure 4: Distance buffers around existing settlements (hatched polygons). White areas are excluded from further suitability considerations. 
The values of the raw material thickness range from 0 to about 42.5 meters. This value span was linearly divided into 7 classes. Since high deposit thicknesses are favorable for a profitable exploitation, areas of high thickness values received higher class values (figure 5). The thickness of the overburden material (maximum value of about $57 \mathrm{~m}$ ) was treated the other way around: The lower the overburden thickness is the more economical is the exploitation of the deposit. Areas of low overburden thicknesses were therefore assigned with high class values (figure 6).

Sand and gravel mining is strictly forbidden in areas of a high water protection level (zones I and II). In principal, it is allowed within water protection zone III (which is further divided into zones IIIa and IIIb). However, sand and gravel mining would be even more preferable in areas with no water protection at all. For the latter (and best) case, a class value of 6 was assigned. Water protection zone IIIb obtained a class value of 4, zone III and IIIa respectively a value of 2 . Zones I and II were excluded from further considerations (figure 7).

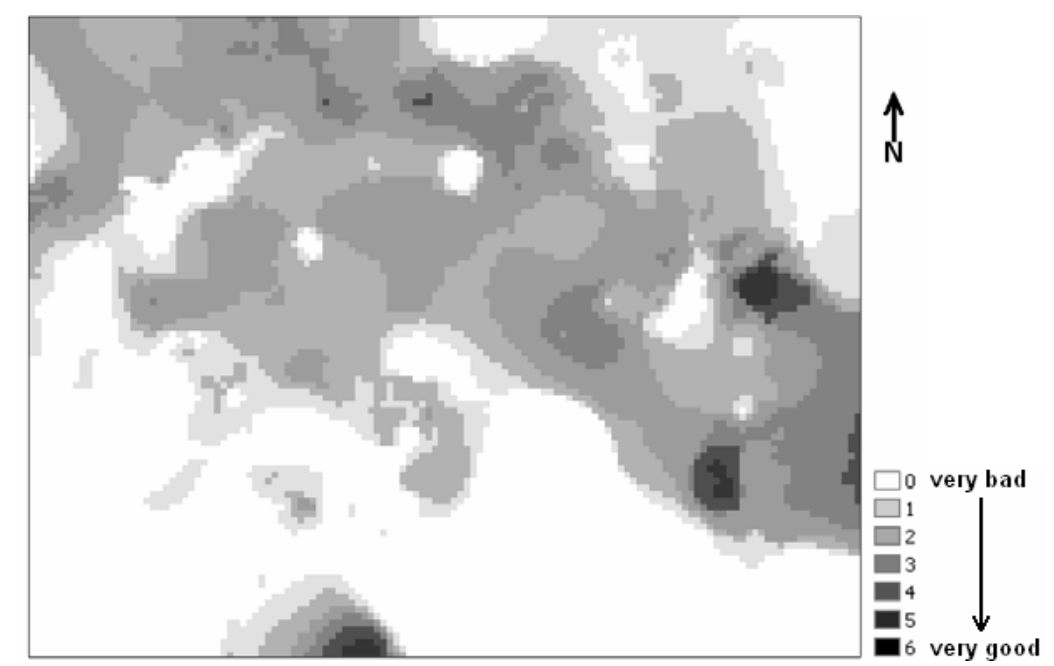

Figure 5: Class values for the thicknesses of sand and gravel deposits. The darker the grey the higher the deposit thickness. White areas are excluded from further suitability considerations.

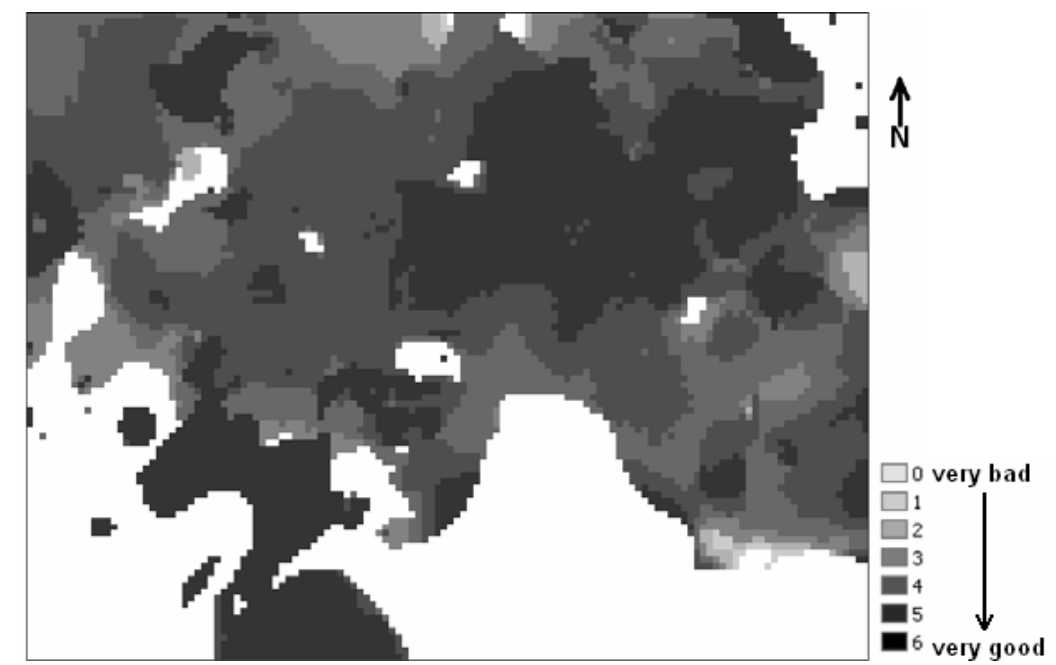

Figure 6: Class values for the thicknesses of the overburden. The darker the grey the lower the overburden thickness. White areas are excluded from further suitability considerations. 




Figure 7: Class values for water protection zones. The higher the class values the better. White areas are excluded from further suitability considerations.

\subsection{Assigning criteria weights}

The weights for the defined criteria were developed on basis of the well known numerical scale for preference values ranging from 1 to 9 (Saaty 1977). However, the criteria weights have the strongest impact on the results and hence the determination of the preference values is often subject to debate among the interest groups involved. To avoid these debates, one should allow each interest group to establish a matrix of preference values of its own. In the presented case study, two preference matrices for two suitability scenarios were established (tables 2 and 3).

Table 2: Preference values for suitability scenario $1^{*}$.

\begin{tabular}{cccccc}
\hline \hline & wpz & dist & overburden & thickness & soil prod. \\
\hline wpz & 1 & 0.25 & 5 & 5 & 0.5 \\
dist & 4 & 1 & 7 & 7 & 4 \\
overburden & 0.2 & 0.143 & 1 & 0.25 & 0.2 \\
thickness & 0.2 & 0.143 & 4 & 1 & 0.143 \\
soil prod. & 2 & 0.25 & 5 & 7 & 1 \\
\hline \hline
\end{tabular}

* wpz: water protection zone [-]; dist: distance to settlements [m]; overburden: thickness of overburden [m]; thickness: thickness of raw material [m]; soil prod.: agricultural productivity of soils [-]

Table 3: Preference values for suitability scenario 2.

\begin{tabular}{cccccc}
\hline \hline & wpz & dist & overburden & thickness & soil prod. \\
\hline wpz & 1 & 5 & 2 & 2 & 4 \\
dist & 0.2 & 1 & 2 & 2 & 4 \\
overburden & 0.5 & 0.5 & 1 & 0.3333 & 4 \\
thickness & 0.5 & 0.5 & 3 & 1 & 4 \\
soil prod. & 0.25 & 0.25 & 0.25 & 0.25 & 1 \\
\hline \hline
\end{tabular}

The values of table 2 reflect the domination of non-economic criteria over economic criteria. Values of 6 and 8 in lines 1, 2 and 5 tell us that criteria like "pit position with respect to a water protection area", "distance to settlements" and "soil productivity" are regarded to be much more important than the thicknesses of the raw material and the overburden. The values of table 3, however, reflect a stronger overall balance between the criteria which is expressed through fairly low preference values. However, "position with respect to water protection area" is slightly dominating, whereas "soil productivity" is dominated by the rest of the criteria.

The difference in these "philosophies" is clearly reflected in the derived criteria weights which are given in table 4 . The weights were calculated with a newly developed ArcGIS ${ }^{\mathrm{TM}}$ (Esri 2004) extension which 
has been developed at the Institute for Applied Geosciences of the Technische Universität Darmstadt. To download this extension, please refer to http://arcscripts.esri.com/.

Table 4:Criteria weights derived from the preference values given in tables 2 and 3.

\begin{tabular}{ccc}
\hline \hline criterion & weights according to table 2 & weights according to table 3 \\
\hline wpz & 0.163 & 0.4151 \\
dist & 0.503 & 0.2067 \\
overburden & 0.037 & 0.1282 \\
thickness & 0.063 & 0.198 \\
soil prod. & 0.241 & 0.052 \\
\hline Consistency ratio CR & 0.123 & 0.125 \\
\hline \hline
\end{tabular}

Please, note that the consistency ratios CR for the derived weights are above 0.1 which - according to Saaty (1977) - would require a revision of the preference matrix. However, the threshold value of 0.1 for the consistency ratio is derived from expert judgement and experience (Saaty 1991) and should therefore not be a formal constraint. Moreover, a CR of about 0.12 is only slightly above the recommended value of 0.1 and the derived weights from tables 2 and 3 plausibly reflect the assigned preference values. Therefore, the preference matrices were not revised and introduced as weights for the subsequent weighted criteria summation.

\subsection{Resulting suitability maps}

Figure 8 shows the suitability map with the weighting factors derived from table 2. Please, note that due to the high weight for the criterion "distance to settlements" the distance buffers can still be well observed in the suitability map. Please, additionally note that the strictly numerical classification has been turned to better understandable alphanumeric suitability descriptors. The class "extremely bad to very bad" coincides with class values 0 to 1 , whereas "very good to excellent" coincides with class values 5 to 6 .

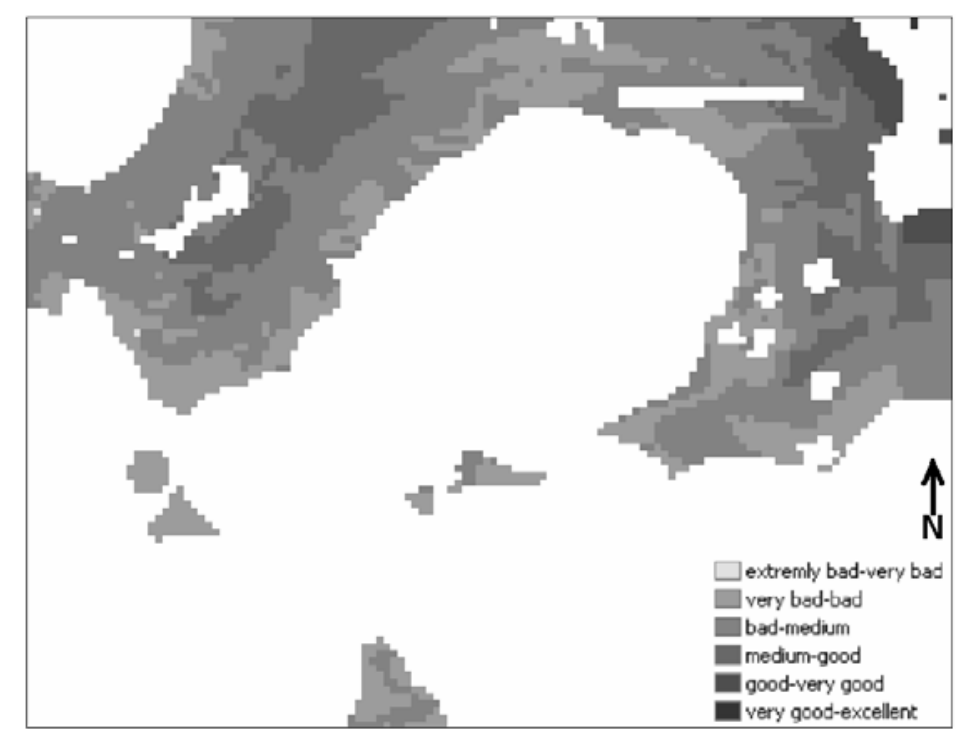

Figure 8: Suitability map derived from the weights which were developed from table 2 . White areas were excluded from suitability considerations (see text).

According to the second set of criteria weights, a second suitability map was calculated. The resulting map is shown in figure 9 .

Since there are still numerical values "behind" the calculated suitability maps, we can calculate a map depicting the differences. This difference map can be interpreted as a map of conflict, since we can assume that we will not have conflicts in regions of the map, where both underlying maps led to the same or at least to similar results. The smaller the difference for a region is, the smaller is the potential for conflict and vice versa. The resulting map is shown in figure 10. 


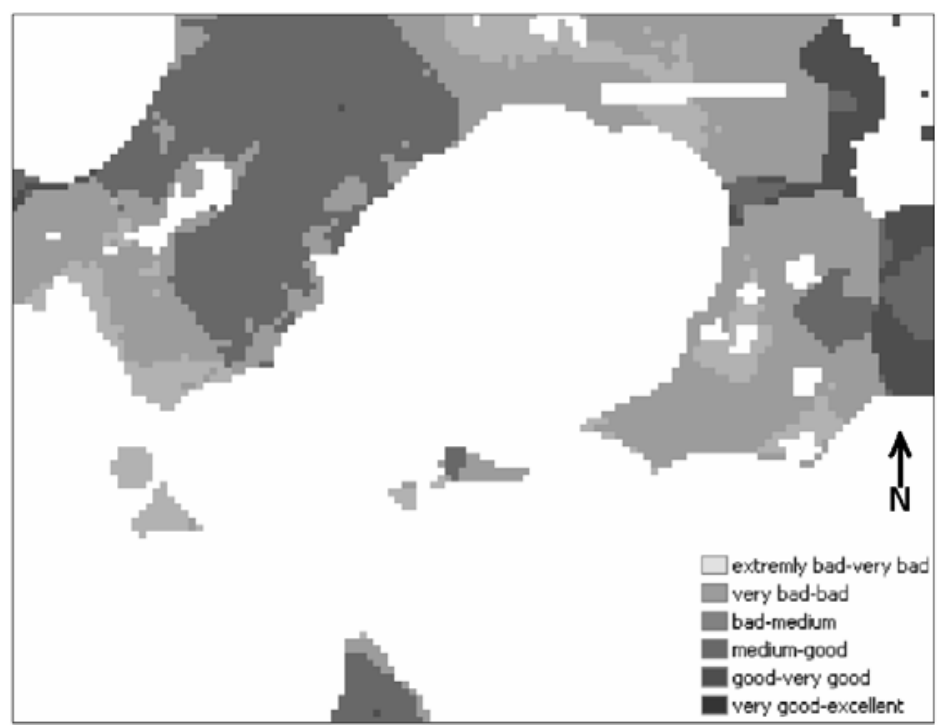

Figure 9: Suitability map derived from the weights which were developed from table 3.

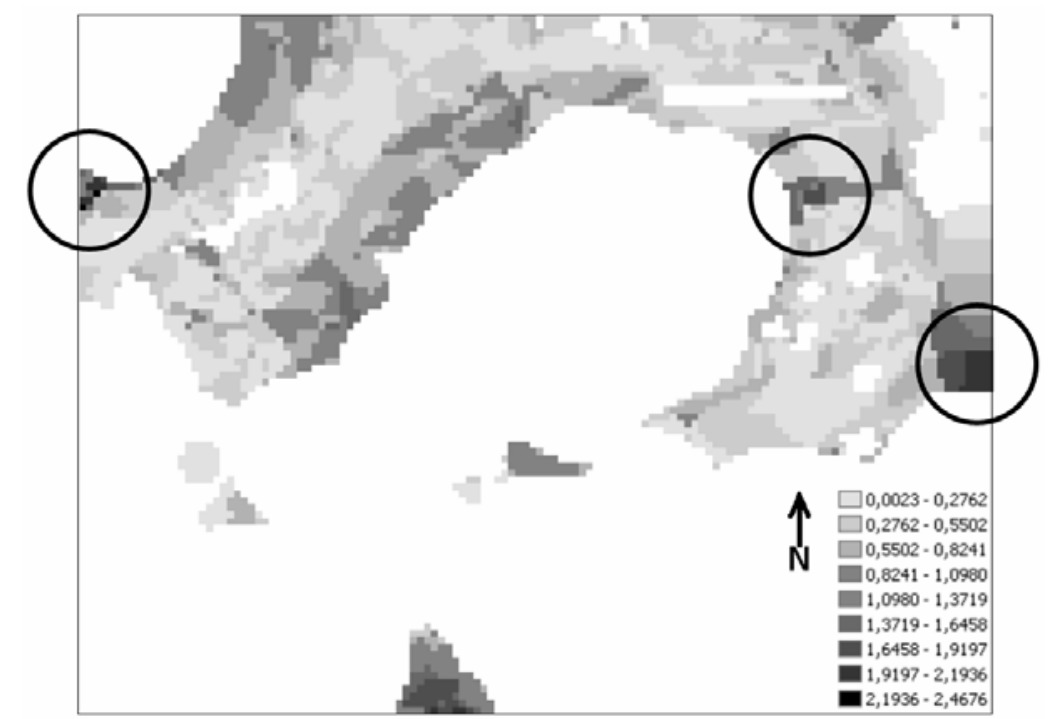

Figure 10: Resulting conflict map. White areas were excluded from suitability considerations. The circles accentuate the areas of highest conflicts.

With the help of figure 10, land-use planners can now clearly distinguish between areas of high and low interest group conflict potential and hence can give recommendations for areas for future raw material exploitation.

\section{Conclusions}

It was shown how the AHP was used to derive the suitability and the conflict potential for a specific landuse within a region in a manner that is easily comprehensible. Depending on the number of interest groups involved, the number of evaluated criteria may be considered too small, as for instance no maps of biodiversity were introduced. However, the presented workflow may be regarded as a template to integrate additional criteria and other land-use scenarios. 


\section{References}

Araújo, C., Mecedo, A. (2002), “Multicriteria Geologic Data Analysis for Mineral Favourability Mapping: Application to a Metal Sulphide Mineralized Area, Ribeira Valley Metalogenic Province, Brazil”. Natural Resources Research, 11, 1, 29-43.

Bund für Naturschutz BfN (2001), BfN-Wissenschaftler unterstützen Geo-Tag der Artenvielfalt im Ruhrgebiet, Pressemitteilung 08.06.2001. - in german -

Carver, S. (1991), “Integrating multi-criteria evaluation with geographical systems”. International Journal of Geographical Information Systems, 5, 321-339.

Dai, F.C.; Lee, C.F., Zhang, X.H. (2001), “GIS-based geo-environmental evaluation for urban land-use planning: a case study”. Engineering Geology, 61, 257-271.

Eastman, J.R., Jiang, H., Toledano, J. (1998), Multi-criteria and multi-objective decision making for land allocation using GIS. In Multicriteria Analysis for Land-Use Management (Beinat, E., Nijkamp, P. (Eds): Kluwer Academic Publishers), 227-251.

ESRI INC. (2004), ArcGIS 9. ESRI Inc., Redlands, California.

Malczewski, J. (1999), Spatial multicriteria decision analysis. In Spatial Multicriteria Decision Making And Analysis - A geographic information sciences approach (Thill. J.C. (ed.), New York, N.Y, Ashgate), 11-48.

Marinoni, O. (2004), "Implementation of the analytical hierarchy process with VBA in ArcGIS". Computers and Geosciences, 30, 6, 637-646.

Marinoni, O. (2005), “A stochastic spatial decision support system based on PROMETHEE”. International Journal of Geographical Information Science, 19, 1, 51-68.

Pereira, J.M.C., Duckstein, L. (1993), “A multiple criteria decision-making approach to GIS-based land suitability evaluation”. International Journal of Geographical Information Systems, 7, 407-424.

Saaty, T.L., (1977), “A scaling method for priorities in hierarchical structures”. Journal of Mathematical Psychology, 15, 231-281.

Saaty, T.L., Vargas, L.G., 1991. Prediction, Projection and Forecasting. Kluwer Academic Publishers, Dordrecht.

Tkach, R.J., Simonovic, S.P., (1997), “A New Approach to Multi-criteria Decision Making in Water Resources”. Journal of Geographic Information and Decision Analysis, 1, 25-44. 